outdoor allergens (short ragweed, perennial rye, Alternaria alternata, Bermuda grass, Russian thistle and white oak), and a food allergen (peanut) and a negative control. Positive allergen response was defined as maximal weal diameter $\geq 3 \mathrm{~mm}$ for allergen and $<3 \mathrm{~mm}$ for negative control.

Results Serum levels of $25(\mathrm{OH}) \mathrm{D}$ were positively associated with FVC in adolescents (0.073 (0.025-0.121) SD) and adults $(0.045$ (0.013-0.078) SD) and with $\mathrm{FEV}_{1}$ in adults (0.050 (0.013-0.087)) after adjusting for health status, previously diagnosed respiratory/ allergic diseases, supplement use, household pets and demographic and socioeconomic characteristics. These associations were independent of circulating calcium levels. Calcium levels were not associated with lung function in either age group. In adults, modest positive associations of ionised calcium with sensitisation to allergens of grass origin were observed (OR per SD, 1.12(1.00-1.26) for white oak and 1.13(1.01-1.26) for Bermuda grass in fully adjusted model). These associations were independent of $25(\mathrm{OH}) \mathrm{D}$ levels. All associations remained after further adjustment for smoking and alcohol use.

Conclusion We found a cross-sectional association between serum 25 $(\mathrm{OH}) \mathrm{D}$ levels and lung function, which does not appear to be driven by increased allergen sensitisation. By contrast we report a novel positive association between serum levels of ionised calcium and grass allergen sensitisation in adults, which is independent of vitamin $\mathrm{D}$

\section{P68 THE PREVALENCE OF EYE DISEASE IN NORFOLK AND WAVENEY}

doi:10.1136/jech.2010.120477.68

A Ighomereho, N Steel, M Bachmann. School of Medicine, University of East Anglia, Norwich, UK

Introduction Visual impairment is an important preventable cause of disability in the UK. Cataract, glaucoma, diabetic retinopathy and age related macular degeneration are the common causes of visual impairment in the blind register, but little is known about the prevalence of eye disease in the community. This study aims to estimate the community prevalence of eye disease.

Methods Twenty-five general practices in Norfolk and Waveney were invited, and seven practices from rural, urban and inner city areas agreed to participate. Anonymised data about age, sex, Read codes, ophthalmic prescription, and post codes were extracted from electronic records using MIQUEST data extraction programme. Patients with an ophthalmic diagnosis between 1st May 2008 and 30th April 2009 were identified. The prevalence of both minor eye conditions such as conjunctivitis and eyelid conditions, and major conditions leading to visual impairment (cataract, glaucoma, diabetic retinopathy and age related macular degeneration) was estimated.

Results 3089 (5.1\%) people with ages ranging from 0 to 104 years (mean 49.9 years) had an eye condition, out of a total practice population of 60739 had at least one eye condition. Of these 3089 people, 1707 (55.3\%) were female, 1382 (44.7\%) were male. 150 $(4.9 \%)$ of these had age related macular degeneration, $200(6.5 \%)$ had glaucoma, $223(7.2 \%)$ had cataract, 371 (12\%) had diabetic retinopathy, 560 (18.1\%) had eyelid conditions, 1211 (39.2\%) had conjunctivitis and benign conjunctival conditions. Some patients had more than one eye condition. The overall prevalence of eye conditions was $5.1 \%$. Prevalence for individual eye conditions were as follows: Age related macular degeneration was $0.2 \%$, Cataract was $0.4 \%$, Diabetic retinopathy was $0.6 \%$, glaucoma was $0.3 \%$, conjunctivitis including benign conjunctival conditions was $2 \%$ and eyelid conditions was $0.9 \%$. A limitation of the study is that we relied on Read codes for identification of eye conditions. Eye conditions may not have been coded at all, and any errors in coding could have introduced misclassification bias.

Conclusions The four eye conditions that are the major causes of preventable visual impairment are frequently encountered in general practice. Further research is needed into the management of these conditions in primary care, so that ways to further reduce avoidable visual impairment can be identified.

\section{P69 HOW TO STOP DRUG USERS DYING FROM AN OVERDOSE: A SYSTEMATIC REVIEW OF TREATMENT AND PREVENTION INTERVENTIONS IN THE COMMUNITY}

doi:10.1136/jech.2010.120477.69

${ }^{1} \mathrm{~A}$ Evans, ${ }^{1} \mathrm{H}$ Snooks, ${ }^{1} \mathrm{D}$ Russell, ${ }^{2} \mathrm{C}$ Brown, ${ }^{2} \mathrm{~A}$ Nair, ${ }^{3} \mathrm{C}$ Moore, ${ }^{4} \mathrm{~A}$ Lewis. ${ }^{1}$ School of Medicine, Swansea University, Swansea, UK: ${ }^{2} A B M$ University NHS Trust, UK: ${ }^{3}$ Welsh Ambulance Service Trust, UK; ${ }^{4}$ Welsh Assembly Government, UK

Objective To describe interventions to prevent and treat overdoses in the pre-hospital setting and review their effectiveness.

Design This review was undertaken in three stages: 1: systematic overview-systematic literature search for all reports of interventions to treat and prevent overdose in the pre-hospital setting; 2 : systematic review-we selected comparative studies from the overview results and undertook qualitative synthesis; 3: we identified studies suitable for meta-analysis.

Setting We looked for interventions providing overdose reversal and/ or prevention treatment in the prehospital emergency setting. UK opiate overdoses are amongst the highest in Europe. People who suffer a non-fatal poisoning are at high risk of death within the following year. For every death there are approximately 7 "near misses". Naloxone is administered to reverse overdose.

Participants Drug users who overdose or are at risk of further overdose.

Main Outcome Measure Prevention of fatal overdose.

Results (1) Systematic overview-39 references described interventions in six categories: 1 . take-home naloxone administered by peers to an overdose patient, following training; 2. CPR training for bystanders witnessing an overdose; 3 . alternative routes of naloxone administration by health professionals(intranasal, subcutaneous, intramuscular, intravenous); 4. police attendance protocols to encourage 999 calls by peers witnessing overdose; 5. supervised injection facilities; 6. psychosocial/educational interventions. (2) Systematic review -15 studies were included. Populations, interventions, methods and outcome were heterogeneous but 10/15 contained at least one comparative result. Quality assessment scores identified 3/15 reasonable quality studies (1 RCT) but most were poor quality. Evidence of effectiveness was weak but suggested death rates may be reduced by: take-home naloxone; bystander CPR; treatment for addiction; naloxone implants. Inter-study results were not comparable. (3) Meta-analysis-we could not identify comparable results across such diverse studies. Meta-analysis of effectiveness was therefore not possible.

Conclusion There is little evidence of effectiveness for interventions identified in this review, including alternative pathways for naloxone administration, although naloxone is an effective treatment to reverse opiate overdose. Rigorous evaluation is needed to assess clinical and cost effectiveness, adverse event rates and effects on drug-taking behaviour of such approaches. 